\title{
Cell Growth Regulation
}

National Cancer Institute

\section{Source}

National Cancer Institute. Cell Growth Regulation. NCI Thesaurus. Code C18080.

Cell growth regulation controls the rate, or the mechanism, of complex coordinated cellular phenomena that promote cell metabolism, cell growth, and cell division. 